\title{
O CUIDADO COMO OBJETO DE PESQUISA NA PRODUÇÃO DE CONHECIMENTO SOBRE POLÍTICAS PÚBLICAS PARA O ENVELHECIMENTO.
}

THE CARE AS RESEARCH SUBJECT IN THE KNOWLEDGE PRODUCTION ON PUBLIC POLICY FOR AGING.

LE SOIN COMME OBJET DE RECHERCHE EN LA PRODUCTIONDE CONNAISSANCES SUR LES POLITIQUES PUBLIQUES DE VIEILLISSEMENT.

EL CUIDADO COMO OBJETO DE INVESTIGACIÓN EN LA PRODUCCIÓN DE CONOCIMENTO SOBRE POLÍTICAS PÚBLICAS PARA EL ENVEJECIMIENTO.

Marisete Teresinha Hoffmann-Horochovski*

ID : https://orcid.org/0000-0002-5220-3614

Maria Tarcisa Silva Bega**

ID: https://orcid.org/0000-0003-4330-8454

Rodrigo Rossi Horochovski***

ID : https://orcid.org/0000-0003-1135-0543

Ingrid Schwyzer****

ID: https://orcid.org/0000-0002-3970-7391

\footnotetext{
* Doutora em Sociologia; Professora do Programa de Pós-Graduação em Desenvolvimento Territorial Sustentável e do Mestrado Profissional em Sociologia da Universidade Federal do Paraná (UFPR), Campus Litoral, Matinhos, PR, Brasil; e-mail: marisetehh@gmail.com

** Doutora em Sociologia; Professora do Departamento de Sociologia, do Programa de Pós-Graduação em Sociologia e do Mestrado Profissional de Sociologia da Universidade Federal do Paraná (UFPR), Curitiba, PR, Brasil; e-mail: tarcisa.silva@gmail.com

*** Doutor em Sociologia Política; Professor dos Programas de Pós-Graduação em Ciência Política e Desenvolvimento Territorial Sustentável da Universidade Federal do Paraná (UFPR), Campus Litoral, Matinhos, PR, Brasil; e-mail: rodrigoh33@gmail.com

**** Doutora em Sociologia; Professora do Centro Universitário Unifacear, Campus Araucária, Araucária, PR, Brasil; e-mail: ingrid.sc@gmail.com
} 
RESUMO: Este artigo analisa o cuidado na produção de conhecimento sobre políticas públicas direcionadas ao envelhecimento no Brasil, por meio de um exercício bibliométrico. Foram selecionados artigos publicados na coleção de periódicos da biblioteca eletrônica SciELO, no período 1994-2017. Os dados referem-se a 29 artigos, escritos por 85 autores e publicados em 15 periódicos distribuídos em três grandes áreas de conhecimento - Ciências da Saúde, Ciências Humanas e Ciências Sociais Aplicadas - e qualificados na área de Sociologia na Capes. Como resultado, observa-se que a maioria das publicações está em revistas da área da Saúde e resulta de pesquisas teórico-empíricas, realizadas principalmente por mulheres, nas temáticas: cuidado em Instituições de Longa Permanência, cuidado em Enfermagem, cuidado/cuidador familiar, ampliação do número de cuidadores, invisibilidade de idosos e cuidadores, cuidado no final da vida e autocuidado. $\mathrm{Na}$ conclusão, destaca-se a feminização da velhice, do cuidado e da produção de conhecimento que aborda o cuidado, bem como a importância de políticas públicas efetivas no envelhecimento com qualidade.

Palavras-chave: cuidado; envelhecimento; bibliometria; produção de conhecimento; políticas públicas.

ABSTRACT: This article analyzes the care in knowledge production on public policies for aging, through a bibliometric exercise. It was selected articles published in journals collection of SciELO electronic library, from 1994 to 2017. The data are related to 29 articles, written by 85 authors and published in 15 journals distributed in three areas of knowledge (Health Sciences, Human Sciences, and Applied Social Sciences) and qualified in Sociology area in CAPES. As a result, most of the publications are in the Health area and result from theoretical-empirical investigations, carried out by women, in the following themes: care in the long-term institutions, care in Nursery, family care/caregivers, increasing in the number of caregivers, invisibility of the elderly and caregivers, and care at the end of life and self-care. In conclusion, we stress the feminization of aging, care, and knowledge production on care as well as the importance of effective public policies for aging with quality.

Keywords: care; aging; bibliometry; knowledge production; public policies.

RESUMÉ: Cet article analyse le soin apporté à la production de connaissances sur les politiques publiques visant le vieillissement au Brésil, à travers un exercice bibliométrique. Les articles publiés dans la collection de revues de la bibliothèque électronique 
O cuidado como objeto de pesquisa na produção de conhecimento sobre políticas públicas para o envelhecimento.

SciELO ont été sélectionnés pour la période 1994-2017. Les données se réfèrent à 29 articles, rédigés par 85 auteurs et publiés dans 15 journaux répartis dans trois grands domaines de la connaissance - sciences de la santé, sciences humaines et sciences sociales appliquées - et qualifiés dans le domaine de la sociologie à Capes. En conséquence, on constate que la plupart des publications sont dans des revues du domaine de la santé et résultent de recherches théoriques et empiriques menées principalement par des femmes dans les domaines suivants: soins dans des établissements de longue durée, soins en soins infirmiers, soins en famille, augmentation du nombre de soignants, invisibilité des personnes âgées et des soignants, soins en fin de vie et soins personnels. En conclusion, se démarquent la féminisation de la vieillesse, les soins et la production de connaissances qui traitent des soins, ainsi que l'importance de politiques publiques efficaces pour un vieillissement de qualité.

Mots-clés: soins; vieillissement; bibliométrie; production de connaissances; politiques publiques.

RESUMEN: Este artículo analiza el cuidado en la producción de conocimiento sobre políticas públicas dirigidas al envejecimiento en Brasil, mediante un ejercicio bibliometrico. Se seleccionaron artículos publicados en la colección de revistas de la biblioteca electrónica SciELO, en el periodo 1994-2017. Los datos se refieren a 29 artículos, escritos por 85 autores y publicados en 15 periódicos distribuidos en tres grandes áreas de conocimiento - Ciencias de la Salud, Ciencias Humanas y Ciencias Sociales Aplicadas - y calificados en el área de Sociología en la Capes. Como resultado, se observa que la mayoría de las publicaciones están en revistas del área de la Salud y resulta de investigaciones teóricas y empíricas, realizadas principalmente por mujeres, en las temáticas: cuidado en Instituciones de Larga Permanencia, cuidado en Enfermería, cuidado/cuidador familiar, ampliación del número de cuidadores, invisibilidad de ancianos y cuidadores, cuidado al final de la vida y autocuidado. En la conclusión, se destaca la feminización de la vejez, del cuidado y de la producción de conocimiento que aborda el cuidado, así como la importancia de políticas públicas efectivas para el envejecimiento con calidad.

Palabras clave: cuidado; envejecimiento; bibliometría; producción de conocimiento; políticas públicas. 


\section{INTRODUÇÃO}

O envelhecimento populacional é um fato concreto no Brasil e no mundo. O Fundo de População das Nações Unidas projeta que a população de idosos será de dois bilhões em 2050, o que corresponde a 22\% da população global (UNFPA, 2012). O Instituto Brasileiro de Geografia e Estatística informa que o percentual de idosos passa de 6,4\% no início dos anos 1980 para 13,75\% em 2014 (IBGE, 2015) e que a média da expectativa de vida cresceu 8,9 nos últimos 25 anos no Brasil, alcançando 75,8 anos em 2016 (IBGE, 2017). Esses dados, por um lado, celebram a vida na medida em que a longevidade representa uma das "maiores conquistas da humanidade" e, por outro, impõem inúmeros desafios que precisam ser superados para que as pessoas possam "envelhecer com dignidade e segurança" (UNFPA, 2012, p.3).

As primeiras preocupações acerca da velhice remontam aos anos 1970 quando germinam debates acadêmicos e políticas públicas, intensificados nas décadas seguintes, que trazem visibilidade para a temática (Debert, 1999). Em 1982 foi realizada a Assembleia Mundial sobre o Envelhecimento na cidade de Viena, convocada pela Assembleia Geral das Nações Unidas em 1978. A Assembleia de 1982 produziu o Plano de Ação Internacional de Viena sobre o Envelhecimento, com 66 recomendações que objetivavam, entre outras, tornar o idoso um ator social, com autonomia e independência financeira (Camarano; Pasinato, 2004). Isso reverberou na Constituição Federal do Brasil de 1988, que introduz a seguridade social (saúde, assistência e previdência social) e determina no artigo 230: "A família, a sociedade e o Estado têm o dever de amparar as pessoas idosas, assegurando sua participação na comunidade, defendendo sua dignidade e bem-estar e garantindo-lhes o direito à vida" (Brasil, 1988).

A Política Nacional do Idoso - PNI (Lei 8.842/1994), regulamentada pelo Decreto 1.948 (1996), é um marco na legislação sobre o envelhecimento no Brasil. Em consonância com a Assembleia de Viena e com a Proclamação do Envelhecimento (1992), a PNI visa assegurar autonomia, direitos sociais e participação dos idosos, in- 
clusive na elaboração de políticas públicas. Não obstante, dificuldades na sua implementação e na efetivação dos direitos dos idosos levaram à criação do Estatuto do Idoso (Lei 10.741/2003), que reúne algumas políticas e leis já aprovadas (Camarano; Pasinato, 2004; Camarano, 2013) e adiciona outras várias, incorporando a integralidade e a equidade e objetivando a promoção do bem-estar na velhice. Em seu artigo terceiro, o Estatuto estabelece que cabe ao Estado garantir ao idoso com prioridade, em conjunto com a família e a sociedade, "a efetivação do direito à vida, à saúde, à alimentação, à educação, à cultura, ao esporte, ao lazer, ao trabalho, à cidadania, à liberdade, à dignidade, ao respeito e à convivência familiar e comunitária" (Brasil, 2003).

Com o Estatuto do Idoso, o Estado reforça sua corresponsabilidade na construção de um envelhecimento ativo, seguindo o conselho do Plano de Ação da Assembleia Mundial sobre o Envelhecimento, realizada em Madri no ano de 2002 (Camarano, 2013), e o registra de forma definitiva na agenda governamental. Políticas de saúde, trabalho e renda, educação, assistência e previdência, se tornam imprescindíveis para o envelhecimento ativo, conforme preconizado pela Organização Mundial de Saúde - OMS (WHO, 2005). Legalmente, o Brasil assegura participação e direitos sociais aos idosos e se prepara para o envelhecimento. Mas na prática cotidiana essa preparação é passível de questionamento.

Diante disso, este artigo objetiva investigar mais sobre o envelhecimento no Brasil, principalmente no que tange à produção de conhecimento sobre as políticas públicas voltadas para a temática, tendo como recorte o cuidado. Ou mais especificamente, interessa averiguar como o cuidado é trabalhado na produção de conhecimento sobre políticas públicas voltadas para o envelhecimento populacional disponibilizada na coleção da biblioteca de periódicos do SciELO (Scientific Eletronic Library Online) e avaliados com extrato superior em Sociologia (A1, A2 e B1) pela Coordenação de Aperfeiçoamento de Pessoal de Nível Superior - Capes.

Ressalta-se que o recorte da pesquisa ocorreu por duas razões fundamentais, que estão entrelaçadas: o cuidado - institucionalizado, domiciliar, profissional, familiar, no final da vida e autocuidado 
- está relacionado ao contexto sócio-histórico de cada sociedade e é essencial ao processo de envelhecimento, devendo ser considerado na elaboração das políticas públicas. Embora haja predomínio de pesquisas em políticas de Saúde, notadamente em Enfermagem, sua complexidade e importância o tornaram objeto de diversas áreas do conhecimento. Nesse sentido, interessa saber se as Ciências Sociais também estão preocupadas com esta questão de extrema relevância para a sociedade e Estado, diante da já aludida redefinição demográfica, e se a inclui em suas análises de políticas. Para tanto, optou-se em fazer um exercício bibliométrico para averiguar quem produz, o que produz, quem publica e quando, com o intuito de traçar um panorama geral do cuidado na produção do conhecimento sobre políticas públicas direcionadas ao envelhecimento no Brasil.

$\mathrm{O}$ artigo está dividido em três partes, além dessa introdução. Na primeira, aborda-se brevemente a conceituação sobre o cuidado. Em seguida, apresenta-se a metodologia que possibilitou a presente pesquisa para, na sequência, apresentar o cuidado na produção de conhecimento sobre envelhecimento, disponibilizada em periódicos do SciELO altamente qualificados em Sociologia. Por fim, traça-se as considerações finais.

\section{SOBRE O CUIDADO}

O cuidado pode ser considerado, ao mesmo tempo, uma prática, uma atitude e mesmo uma disposição moral. Historicamente relacionado ao espaço doméstico, o cuidado também caracteriza determinados campos profissionais como o da saúde e do trabalho social. No caso dos referidos campos, constata-se, ainda, a concepção do cuidado como um trabalho essencialmente feminino. $\mathrm{O}$ cuidar foi interpretado, em um primeiro momento, como um "trabalho por amor e vocação", em especial das mulheres. Segundo Tronto (1997), as atividades de cuidar ainda são regidas pelas questões de gênero tanto no âmbito do mercado de trabalho, quanto no espaço privado. Exemplo é a Enfermagem, cuja a identidade profissional é construída em torno do cuidado, da mesma maneira que agrega um maior número de mulheres que homens em suas funções. No entanto, Collière 
O cuidado como objeto de pesquisa na produção de conhecimento sobre políticas públicas para o envelhecimento.

(1999) e Leiniger (2000) alertam que não se pode considerar a existência de apenas um cuidado. Afinal, ele pode expressar-se através de diversas ações, modos de vida, valores e nas formas como as práticas do cuidar são simbolicamente representadas pelos indivíduos e pela coletividade.

Leininger (2000) resgata o cuidado como uma prática universal, existente em todas as culturas, e não uma ação ligada especificamente à uma profissão. Em sua ótica, em qualquer cultura, os seres humanos percebem e experimentam comportamentos relativos ao cuidar e ao não cuidar dentro da família e do grupo. "Assim, culturas diferentes percebem, conhecem e praticam o cuidado de maneiras diferentes, apesar de haver pontos comuns no cuidado de todas as culturas no mundo" (Leininger, 2000, p. 298). Nesta perspectiva, não existe apenas um cuidado, mas sim determinadas ações de cuidar, que não se constroem em um vazio, mas que se conectam ao meio histórico e sociocultural em que estão inseridas. As práticas do cuidar são parte da totalidade do social.

O cuidado é, então, próprio do fazer humano e está relacionado com as dimensões da vida e com o contexto de cada sociedade. $O$ cuidar transcende a técnica, de acordo com Waldow (2006), pois traz consigo outras situações como a cultura, as relações sociais e as emoções.

Uma das mais antigas práticas da humanidade, o cuidado visa a continuação da vida e, por extensão, a luta contra a morte. Conceito polissêmico, deriva do latim cura (coera), pode ser utilizado para expressar uma atitude de desvelo e preocupação por uma pessoa ou mesmo um objeto. Todo indivíduo, em algum momento de sua existência, cuidará e será cuidado (Boff, 2012). Juntamente com o trabalho, o cuidado possibilita ao ser humano a construção de seu próprio ser e da sua relação com o outro e com o mundo. É constitutivo da condição humana, fornecendo subsídios para vida social.

Na linguagem cotidiana, cuidado revela uma imensidão de sentimentos, ações e atividades, apesar de sempre implicar algum tipo de responsabilidade e compromisso contínuo. Para Tronto (1997, p. 188), os cuidadores estão "dispostos a trabalhar, a se sacrificar [...] a mostrar envolvimento emocional e a despender energia em relação ao objeto de cuidados". Cuidar é uma ação relacional. A autora, com 
base no objeto do cuidado, distingue "cuidado com" de "cuidar de". Enquanto o primeiro refere-se ao compromisso com cuidar de forma geral, o segundo diz respeito a atender determinadas necessidades específicas (espirituais, físicas, materiais e/ou emocionais) de outro.

A ação de cuidar sempre necessita do outro. Por meio dela, "o eu e o tu transcendem a barreira que os separa e se constrói um nós. Esse nós resultante não implica a dissolução do eu no tu, mas a integração de ambos no sentido de construção". O cuidar implica "abrir-se à perspectiva do nós" (Roselló, 2009, p.128). Essa perspectiva demonstra que a ação de cuidar é um exercício de alteridade e em cada fase da vida humana assume um significado diferente.

$\mathrm{O}$ cuidar remete, portanto, à criação de um vínculo e um compromisso com o outro, independentemente de quem ele seja ou da durabilidade temporal. Cuidar promove a tessitura de laços que podem ser afetivos, profissionais, sociais ou morais. Para Hirata e Guimarães (2012), o cuidado relaciona-se tanto a uma atitude quanto a uma ação. Dessa maneira, o cuidado circula de diferente formas e sentidos na vida social. Sempre existe o outro, seja o objeto do cuidado, seja aquele que o pratica. Neste círculo de trocas, destaca-se a criação, manutenção e mesmo a regeneração dos vínculos sociais.

$\mathrm{Na}$ velhice o cuidado é central e pode ocorrer de diferentes formas: profissional e/ou familiar, institucionalizado e/ou domiciliar. O envelhecimento saudável e ativo é fruto de práticas de cuidado e de autocuidado, também em outras etapas da vida. E é o cuidado no final da vida que pode garantir um final de vida com maior qualidade e menos sofrimento, propiciando uma boa morte, uma ars moriendi moderna (Gawande, 2015).

A importância do cuidado no processo de envelhecimento é evidenciada no próprio Estatuto do Idoso que, no seu artigo $9^{\circ}$, estabelece que: "É obrigação do Estado garantir à pessoa idosa a proteção à vida e à saúde, mediante efetivação de políticas públicas que permitam um envelhecimento saudável e em condições de dignidade" (Brasil, 2003). Mas isso gera algumas contradições como, por exemplo, a do direito ao acompanhante familiar em tempo integral quando da internação da pessoa idosa em instituições hospitalares. Camarano (2013) alerta que o direito é interpretado como dever em 
hospitais públicos e que o familiar fica sobrecarregado, passando a exercer tarefas de cuidado outrora desenvolvidas por profissionais, como alimentar e dar banho no idoso. Essa sobrecarga da família é também observada na priorização do cuidado do idoso dependente na esfera doméstica. Geralmente "o cuidado recai sobre a mulher, ou seja, desresponsabiliza o Estado da função de cuidar, mas lhe atribui a responsabilidade de fiscalizar e punir. Isto significa desconsiderar as mudanças na família, no papel social da mulher e no envelhecimento da própria população idosa" (Camarano, 2013, p. 21).

Em que pese essas e outras contradições, é inegável, contudo, que o Estatuto e própria PNI propiciaram inúmeros avanços na construção de políticas públicas, notadamente sociais, direcionadas ao idoso. E em inúmeras dessas políticas o cuidado é (ou deveria ser) focalizado. E é justamente isso que este trabalho procura averiguar a partir da análise do cuidado na produção de conhecimento sobre políticas públicas direcionadas ao envelhecimento.

\section{NOTAS METODOLÓGICAS}

O presente trabalho, de caráter descritivo e explicativo, consiste num exercício bibliométrico para averiguar como o cuidado é trabalhado na produção de conhecimento de políticas públicas direcionadas ao envelhecimento no Brasil. Como critério para a seleção dos artigos observou-se a presença na coleção de periódicos do SciELO, especificamente nos avaliados pela Capes (Quadriênio 2013-2016) com extrato superior em Sociologia, no período 1994 ano em que foi promulgada a PNI - a 2017, precisamente novembro, quando foi finalizado o banco de dados. O recorte procurou viabilizar o tratamento dos dados, bem como identificar periódicos de diferentes áreas do conhecimento que recebem contribuições do pensamento sociológico, importante tanto para a análise de políticas públicas quanto para a reflexão do processo de envelhecimento como construção social.

A busca inicial selecionou artigos a partir das palavras envelhecimento, idosos e políticas públicas, propiciando, após um trabalho de garimpagem, um banco de dados de 420 artigos que versam 
sobre políticas públicas voltadas ao envelhecimento. Num segundo momento, identificou-se neste banco todos os artigos que utilizaram o termo cuidado no título, resumo ou palavras-chave, num total de 29 artigos. Apesar do corpus da análise (vide Apêndice) ser relativamente baixo, optou-se por usar a bibliometria para visualizar os dados e levantar questões que propiciem aprofundamentos teóricos e/ou novas pesquisas. Ressalta-se que se o objeto fosse o trabalho do cuidado na velhice, sem o recorte estabelecido neste estudo, o número de artigos seria bem maior e os resultados diferentes. Esse inclusive pode ser objeto de uma nova pesquisa: a produção de conhecimento sobre cuidado na velhice.

É importante dizer que a bibliometria, enquanto técnica quantitativa, visa avaliar a produção e disseminação do conhecimento científico, identificando tendências, autores, periódicos, instituições e palavras que se destacam em determinada área. Logo após a seleção dos artigos, foi realizada a marcação dos seguintes elementos: autor(es), filiação institucional do(s) autor(es), título, periódico, local/estado/região de publicação, ano de publicação, resumo e palavras-chave (Francisco, 2011; Clemente; Juliano, 2013). Elementos que fornecem um panorama geral sobre a produção do conhecimento científico em determinada temática.

A marcação das referências bibliográficas foi feita por meio do RQDA, do pacote R para análise de dados qualitativos; foi considerada apenas uma única menção, caso o mesmo autor ou instituição tenham sido referenciados mais de uma vez no artigo. Todos esses elementos foram organizados em uma planilha do Microsoft Excel para viabilizar o tratamento e a análise de dados. Empregou-se o site wordclouds para marcação da frequência e geração de nuvens de palavras e o software Gephi para análise e visualização de redes das referências bibliográficas, a partir das comunidades formadas. Ressalta-se que, em uma rede, denomina-se comunidades o conjunto de nós (as referências) que apresenta maior proximidade entre si, identificados pela aplicação do algoritmo de modularidade (Blondel et al, 2008). Portanto, no caso de uma rede semântica, trata-se das palavras que tendem a estar mais próximas no(s) texto(s) que compõem o 
corpus analisado, formando conjuntos temáticos. Por fim, a análise de conteúdo (Bardin, 2011) possibilitou a realização de inferências e a interpretação dos dados.

\section{RESULTADOS E DISCUSSÃO}

O ano inicial da busca de artigos, como já dito, foi 1994, mas a primeira publicação é de 2003, ano em que foi promulgado o Estatuto do Idoso. A partir daí, mesmo que sem uniformidade, pode-se observar uma tendência ascendente do cuidado na produção acadêmica sobre políticas públicas voltados ao envelhecimento, conforme pode ser observado no gráfico 1 .

Gráfico 1: Publicações por ano

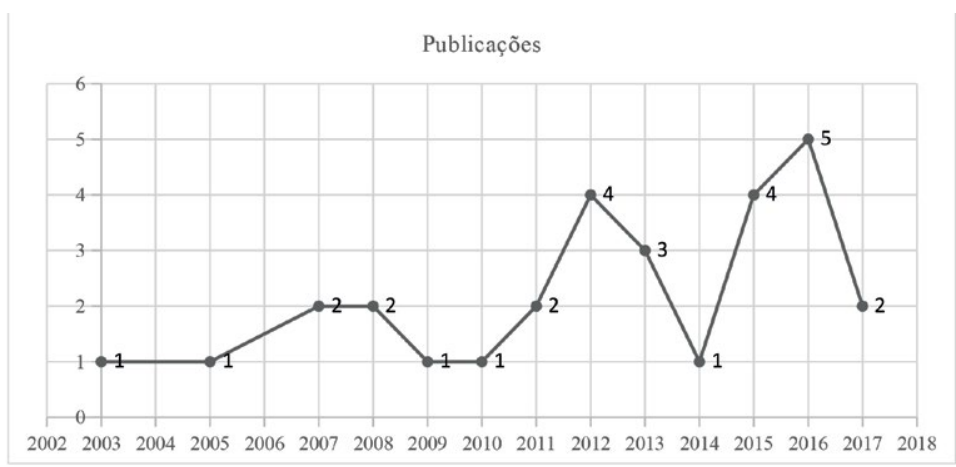

Fonte: Elaboração própria.

O maior número de publicações data de 2016, mas sublinha-se que o número de 2017 não corresponde a todas as edições dos periódicos, posto que o banco foi finalizado no mês de novembro.

A tabela 1 apresenta a distribuição dos artigos por periódicos, considerando sua vinculação institucional e local/região de publicação. 
Tabela 1: Distribuição de artigos por periódico

\begin{tabular}{|c|c|c|c|c|c|}
\hline Periódico & Instituição & $\begin{array}{l}\mathrm{N} \text { ar- } \\
\text { tigos }\end{array}$ & $\begin{array}{l}\text { Local de } \\
\text { Publicação }\end{array}$ & Estado & Região \\
\hline $\begin{array}{l}\text { Revista Brasileira de } \\
\text { Ciência Política }\end{array}$ & UnB & 1 & Brasília & DF & $\mathrm{CO}$ \\
\hline $\begin{array}{l}\text { Psicologia: Ciência e } \\
\text { Profissão }\end{array}$ & CFP & 1 & Brasília & DF & $\mathrm{CO}$ \\
\hline Sociedade e Estado & UnB & 2 & Brasília & DF & $\mathrm{CO}$ \\
\hline $\begin{array}{l}\text { Physis: Revista de } \\
\text { Saúde Coletiva }\end{array}$ & IMS/UERJ & 2 & $\begin{array}{l}\text { Rio de } \\
\text { Janeiro }\end{array}$ & $\begin{array}{l}\text { Rio de } \\
\text { Janeiro }\end{array}$ & SE \\
\hline $\begin{array}{l}\text { Revista Brasileira de } \\
\text { Estudos de População }\end{array}$ & ABEP & 1 & $\begin{array}{l}\text { Rio de } \\
\text { Janeiro }\end{array}$ & $\begin{array}{l}\text { Rio de } \\
\text { Janeiro }\end{array}$ & SE \\
\hline $\begin{array}{l}\text { Cadernos de Saúde } \\
\text { Pública }\end{array}$ & ENSP/Fiocruz & 3 & $\begin{array}{l}\text { Rio de } \\
\text { Janeiro }\end{array}$ & $\begin{array}{l}\text { Rio de } \\
\text { Janeiro }\end{array}$ & SE \\
\hline $\begin{array}{c}\text { Ciência \& Saúde } \\
\text { Coletiva }\end{array}$ & ABRASCO & 5 & $\begin{array}{l}\text { Rio de } \\
\text { Janeiro }\end{array}$ & $\begin{array}{l}\text { Rio de } \\
\text { Janeiro }\end{array}$ & SE \\
\hline $\begin{array}{l}\text { Interface - Comunica- } \\
\text { ção, Saúde, Educação }\end{array}$ & Unesp & 2 & Botucatu & $\begin{array}{l}\text { São } \\
\text { Paulo }\end{array}$ & SE \\
\hline Cadernos Pagu & Unicamp & 3 & Campinas & $\begin{array}{c}\text { São } \\
\text { Paulo }\end{array}$ & $\mathrm{SE}$ \\
\hline Revista CEFAC & ABRAMO & 1 & Campinas & $\begin{array}{c}\text { São } \\
\text { Paulo }\end{array}$ & SE \\
\hline $\begin{array}{l}\text { Revista Latino-Ame- } \\
\text { ricana de Enferma- } \\
\text { gem }\end{array}$ & EERP/USP & 2 & $\begin{array}{l}\text { Ribeirão } \\
\text { Preto }\end{array}$ & $\begin{array}{l}\text { São } \\
\text { Paulo }\end{array}$ & SE \\
\hline Cadernos de Pesquisa & $\begin{array}{c}\text { Fundação } \\
\text { Carlos Chagas }\end{array}$ & 1 & São Paulo & $\begin{array}{l}\text { São } \\
\text { Paulo }\end{array}$ & SE \\
\hline Estudos Avançados & USP & 1 & São Paulo & $\begin{array}{c}\text { São } \\
\text { Paulo }\end{array}$ & SE \\
\hline $\begin{array}{l}\text { Revista da Escola de } \\
\text { Enfermagem da USP }\end{array}$ & USP & 2 & São Paulo & $\begin{array}{l}\text { São } \\
\text { Paulo }\end{array}$ & SE \\
\hline Saúde e Sociedade & USP & 2 & São Paulo & $\begin{array}{c}\text { São } \\
\text { Paulo }\end{array}$ & $\mathrm{SE}$ \\
\hline
\end{tabular}

Fonte: Elaboração própria.

Os quinze periódicos representam doze instituições localizadas em Brasília, Rio de Janeiro, Botucatu, Campinas, São Paulo e Ribeirão Preto. Se considerarmos por região, quatro (13,8\%) publicações são da região Centro-Oeste e 25 (86,2\%), da região Sudeste, sendo $11(37,9 \%)$ do Rio de Janeiro e 14 (48,3\%) de São Paulo. É importante destacar que são três periódicos localizados em Brasília, quatro no Rio de Janeiro e oito no estado de São Paulo. O periódico que concentra mais publicações é o Ciência \& Saúde Coletiva, da 
Associação Brasileira de Saúde Coletiva (ABRASCO), localizado no estado do Rio de Janeiro (5 artigos), e qualificado pela Capes com extrato superior em diferentes áreas do conhecimento.

Oito periódicos associados à área de Ciências da Saúde respondem por $19(65,52 \%)$ artigos, incluindo o já mencionado Ciência \& Saúde Coletiva. Embora quatro deles também constem na lista de Ciências Humanas do SciELO - História, Ciências, Saúde-Manguinhos, Interface-Comunicação, Saúde e Educação, Physis e Saúde e Sociedade - a vinculação institucional os relacionam primeiramente à Saúde. Cadernos de Saúde Pública, Revista CEFAC e Revista da Escola de Enfermagem da USP totalizam a relação. Já as Ciências Humanas respondem por sete $(24,14 \%)$ artigos, publicados nos seguintes periódicos: Revista Brasileira de Ciência Política, Psicologia: Ciência e Profissão, Cadernos Pagu, Cadernos de Pesquisa e Estudos Avançados. Por fim, dois periódicos das Ciências Sociais Aplicadas - Revista Brasileira de Estudos da População e Sociedade e Estado - foram responsáveis por três $(10,34 \%)$ publicações.

Os artigos foram escritos por 85 autores, sendo que seis artigos foram elaborados por um autor, nove por dois autores e 14 por três ou mais. Os artigos assinados por um único autor são predominantemente das Ciências Sociais e Humanas (5) e do Serviço Social (1). A maioria dos artigos escrito em coautoria, 19 precisamente, são relacionados à grande área da Saúde. Uma observação atenta nas revistas da área, permite averiguar que essa é uma prática comum nas discussões da Saúde. Isso é evidenciado em Conner, Provedel e Maciel (2017) que, ao analisar a produção científica na revista Ciência \& Saúde Coletiva, indicam uma média de 3,55 autores por artigo, dentro da tendência atual à superação da produção solitária que caracterizou a produção do conhecimento no passado, especialmente nas Ciências Sociais (Metz; Jäckle, 2017).

A tabela 2 permite visualizar as instituições de origem dos autores dos artigos. 
Tabela 2: Distribuição de autores por instituição de afiliação

\begin{tabular}{|c|c|c|c|}
\hline Instituição de origem & $\begin{array}{l}\mathrm{N} \text { au- } \\
\text { tores }\end{array}$ & Região & País \\
\hline UFRN & 1 & Nordeste & Brasil \\
\hline UFC & 5 & Nordeste & Brasil \\
\hline Faculdade Luciano Feijão CE & 1 & Nordeste & Brasil \\
\hline UnB & 9 & $\begin{array}{l}\text { Centro- } \\
\text {-Oeste }\end{array}$ & Brasil \\
\hline Universidade Católica de Brasília & 2 & $\begin{array}{l}\text { Centro- } \\
\text {-Oeste }\end{array}$ & Brasil \\
\hline PUC Minas & 1 & Sudeste & Brasil \\
\hline UFMG & 14 & Sudeste & Brasil \\
\hline UFJF & 1 & Sudeste & Brasil \\
\hline UNIMONTES & 1 & Sudeste & Brasil \\
\hline USP & 9 & Sudeste & Brasil \\
\hline Unicamp & 1 & Sudeste & Brasil \\
\hline PUC SP & 1 & Sudeste & Brasil \\
\hline Centro Universitário do Norte Paulista & 1 & Sudeste & Brasil \\
\hline ENSP Fiocruz & 7 & Sudeste & Brasil \\
\hline UFSC & 6 & Sul & Brasil \\
\hline UFRGS & 5 & Sul & Brasil \\
\hline Hospital Moinhos de vento & 2 & Sul & Brasil \\
\hline Universidade Tuiuti do Paraná & 3 & Sul & Brasil \\
\hline UEM & 7 & Sul & Brasil \\
\hline Unioeste & 1 & Sul & Brasil \\
\hline $\begin{array}{l}\text { Escola Superior de Enfermagem Cruz Ver- } \\
\text { melha Portuguesa de Oliveira de Azeméis }\end{array}$ & 1 & & Portugal \\
\hline Universidade de Aveiro & 1 & & Portugal \\
\hline Escola Superior de Enfermagem do Porto & 2 & & Portugal \\
\hline University ol Alberta & 1 & & Canadá \\
\hline Universidad Andrés Bello & 1 & & Chile \\
\hline $\begin{array}{l}\text { Universidad ITESO - Universidad Jesuita } \\
\text { de Guadalajara }\end{array}$ & 1 & & México \\
\hline
\end{tabular}

Fonte: Elaboração própria. 
Os 85 autores estão distribuídos em 26 instituições. 78 $(75,86 \%)$ autores são afiliados a 22 instituições distribuídas pelas regiões brasileiras, excetuando o Norte. A região com maior número (46\%) é a Sudeste que, historicamente, concentra importantes instituições de ensino e pesquisa, como é o caso da Universidade Federal de Minas Gerais - UFMG (14 autores), Universidade de São Paulo USP (9) e Escola Nacional de Saúde Pública Sérgio Arouca - ENSP/ Fiocruz (7). Quatro autores (13,79\%) são afiliados a três instituições portuguesas. Um autor $(3,45 \%)$ representa uma instituição do Canadá, outro $(3,45 \%)$ uma do Chile, e um $(3,45 \%)$ terceiro uma do México.

Com relação ao gênero dos autores, $76(89,4 \%)$ são mulheres e nove (10,6\%) são homens. Este dado é deveras importante, porque parece indicar que a feminização da velhice e a feminização do cuidado se estende para a produção de conhecimento sobre políticas públicas direcionadas ao envelhecimento que se preocupam com o cuidado. Não se pode deixar de registrar que boa parte dessa produção está atrelada a campos do conhecimento que integram a grande área Ciências da Saúde e que em alguns deles, notadamente na Enfermagem, a feminização persiste na qualificação profissional (Lopes; Leal, 2005).

No que se refere ao conteúdo dos artigos procurou-se marcar, seguindo Fernandes (2011), os títulos, resumos, palavras-chave e referências bibliográficas de cada um deles. Utilizou-se a ferramenta de frequência de palavras para verificar os termos mais utilizados nos títulos, considerando as mencionadas duas vezes ou mais. Obteve-se os seguintes resultados, observando que não houve distinção 
entre plural e singular, bem como entre feminino e masculino: idoso (22 citações), cuidado (15), cuidador (13), familiar (7), saúde (6), dependente (5) e domiciliar (4), enfermagem (3), instituição de longa permanência (3), perfil (3), percepção (3), qualidade (3), Rio (3), Grande (3), região (3), populacional (2), sobrecarga (2), associados (2), funcional (2), Portugal (2), atenção (2), fatores (2), envelhecimento (2), necessidade (2), prevalência (2), Porto (2) e velho (2).

Com relação às palavras-chave, e inspirado em Clemente e Juliano (2013), verificou-se a frequência dos termos utilizados duas ou mais vezes e foi gerado nuvens de palavras (figura 1) com a utilização do site wordclouds.

Figura 1: Nuvem de palavras dos descritores dos artigos

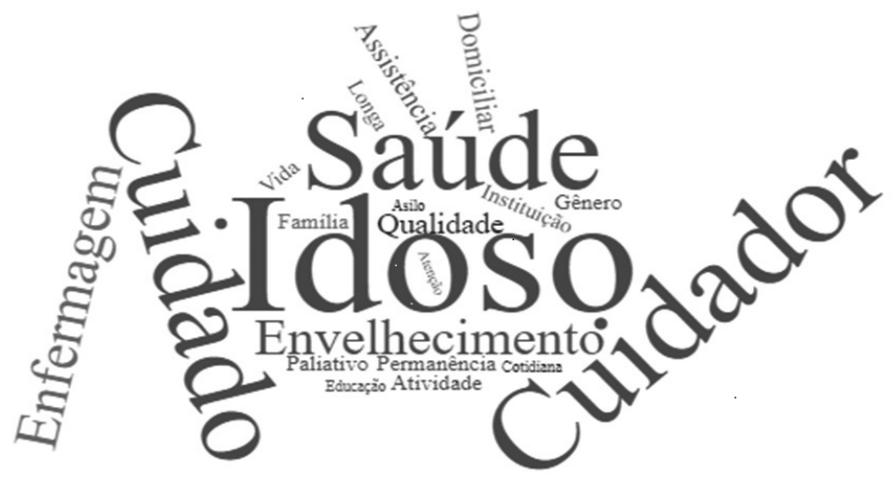

Fonte: Elaboração própria.

Os termos idoso (24 citações), cuidador (17), cuidado (16) e saúde (16) são os mais destacados. Na sequência, estão enfermagem (8) e envelhecimento (6). Domiciliar, assistência e qualidade aparecem quatro vezes cada. Instituição (de) Longa Permanência, atividade, paliativo, família, gênero e vida são mencionadas três vezes. Atenção, cotidiana, educação e asilo são referidas duas vezes.

Os resumos, por sua vez, possibilitaram identificar a abordagem metodológica e os diferentes tipos de cuidados abordados. Dentre os 29 artigos, sete resultam de pesquisa bibliográfica e do- 
cumental e 22 estão embasados em pesquisa teórica-empírica que utilizaram técnicas variadas: entrevistas, etnografia, fhotovoice, questionários SF-36 (Medical Outcomes Study 36-Item Short-Form Health Survey), WHOQOL-Bref (World Health Organization Quality of Life) e QPFC (Questionário de Perfil de Familiar Cuidador).

No que tange ao cuidado propriamente dito, seis artigos abordam o cuidado formal em Instituições de Longa Permanência (ILPs); quatro o cuidado em Enfermagem; dois o cuidado no final da vida; 11 o cuidado domiciliar e o cuidador familiar; três o crescimento do número de cuidadores; dois a (in)visibilidade da díade idoso e cuidador; um o autocuidado. Sequencialmente, procura-se compilar os artigos pesquisados dentro dessas temáticas.

As ILPs têm sido objeto constante de pesquisas sobre envelhecimento e estão presentes em seis estudos que discutem cuidado formal. Batista e Bandeira (2015, p. 80) analisam sociologicamente o trabalho das cuidadoras de mulheres idosas, que recriam técnica corporal que engloba "dimensões afetiva, cognitiva, moral e de poder". Também em uma perspectiva sociológica, Batista e Araújo (2011, p. 175) sublinham a "mercantilização da vida íntima", com a organização taylorista do trabalho instrumentalizando e limitando a ação das cuidadoras, que não é só técnica, mas afetiva e moral. Ambas as discussões reforçam a feminização do cuidado nas instituições de longa permanência e as diferentes dimensões envolvidas no trabalho de cuidar.

Freitas e Noronha (2010) abordam o cuidado profissional nas ILPs e destacam que nem sempre corresponde ao esperado. Consideram que é necessário ampliar os debates, sublinhando tanto a sociabilidade quanto a promoção da saúde do idoso. A saúde bucal de idosos residentes em instituições de longa permanência é analisada por Mello e Erdmann (2007, p. 139) que identificam dois estágios diferenciados: um é de continuidade na "precariedade do quadro epidemiológico" e outro traz modificações, com a incorporação de "melhores práticas de cuidado".

Ribeiro et al (2008) avaliam o perfil de cuidadores em ILPs filantrópicas e privadas, observando que não há diferença significativa quanto ao gênero e remuneração dos cuidadores. Barbosa et al (2017), por sua vez, traçam o perfil sociodemográfico e ocupacional do cuidador de 
idosos em instituições filantrópicas e privadas. As autoras verificam a qualidade de vida dos cuidadores no quesito saúde e concluem que não há disparidade significativa de acordo com a natureza das instituições.

Quatro artigos abordam o cuidado profissional em Enfermagem. Caldeira et al (2012) estudam o cuidado à mulher idosa sob a perspectiva do enfermeiro. Este considera em sua prática tanto os conhecimentos adquiridos, quanto a situação biográfica da paciente, além de conceder à família o papel de intermediação no cuidado. A percepção dos estudantes de Enfermagem e Fonoaudiologia sobre o cuidado na velhice é objeto de Mendes, Soares e Massi (2015). As autoras demonstram que os estudantes consideram que a formação na graduação deixa a desejar no que se refere ao trabalho profissional com idosos. Isto ocorre pois ao longo da graduação a questão referente à saúde do idoso é trabalhada de um modo generalizado dentro da Saúde Coletiva. Apesar da existência da Política Nacional do Idoso e do Estatuto do Idoso, as especificidades da velhice no campo da Enfermagem ainda são pouco discutidas.

As características dos idosos atendidos por uma Equipe de Saúde da Família - ESF, foi alvo da investigação de Meireles et al (2007), que defendem o levantamento de dados para embasar a gestão do cuidado, por meio de políticas efetivas de saúde ao idoso. Carvalhais e Sousa (2013) analisam os fatores relacionados a qualidade dos cuidados domiciliares em enfermagem à idosos dependentes e frisam a importância dos recursos, materiais e humanos, da multidisciplinariedade, da determinação e do apoio aos cuidadores informais.

Dois artigos tratam do cuidado profissional no final da vida. Gomes e Othero (2016) apresentam o estado da arte dos cuidados paliativos no Brasil, que visa o cuidado integral de doentes graves, por meio de prevenção e controle dos sintomas, bem como do apoio à família, cuidador formal e informal e equipe de saúde que sofrem junto. Queiroz et al (2013) refletem sobre os cuidados no final da vida no âmbito da Atenção Primária à Saúde (APS) e concluem que "apesar dos esforços, pouco se logra de melhoria na qualidade de vida de quem morre por uma doença em fase terminal no domicílio, sob os cuidados do Sistema Único de Saúde (SUS) em municípios distantes dos grandes centros" (Queiroz et al, 2013, p.265). 
Onze artigos discutem o cuidado domiciliar exercido por membros da família. Pimenta et al (2009) caracterizam o perfil do cuidador familiar de idosos dependentes na região do Porto, Portugal, e destacam a mulher de aproximadamente 55 anos como a principal cuidadora. Os autores ressaltam que tanto os idosos dependentes quanto seus cuidadores devem ser acolhidos pelos serviços de saúde. Del Duca, Martinez e Bastos (2012) analisam o perfil do cuidador domiciliar de idosos dependentes no sul do Brasil e sublinham que a maioria dos cuidadores são familiares, especialmente filhos/as e cônjuges, o que deve ser considerado nas políticas para idosos. Resende e Dias (2008) reforçam o papel da mulher no cuidado domiciliar e a importância de estudos sobre os cuidadores de idosos, justamente para auxiliar na construção de políticas públicas. Küchemann (2012) corrobora com a ideia de que na prática familiar a mulher se responsabiliza pelo cuidado, mas salienta que o Estado e a sociedade são legalmente corresponsáveis pelo cuidado dos idosos e que um envelhecimento com cidadania exige a participação de todos. Rosas (2014) aborda a feminização do cuidado no México, a partir da perspectiva de mulheres pobres, contempladas por programas sociais. A autora expõe a insuficiência das políticas públicas direcionadas ao envelhecimento e assinala a importância da coletivização do cuidado através da participação de instituições estatais, mercado, sociedade e família. Apesar de estudos e contextos diferenciados, esses artigos apresentam em comum a feminização do cuidado e a necessidade de haver políticas públicas destinadas aos idosos, conforme já sublinhado por Camarano (2013).

Ainda no conjunto de artigos sobre cuidado domiciliar, Israel, Andrade e Teixeira (2011) identificam as percepções dos cuidadores familiares de idosos em condição de dependência física após alta hospitalar e destacam que suas ações objetivam a recuperação integral. Karsch (2003) discute o papel do cuidador familiar na assistência a idosos dependentes por doença crônica e degenerativa e frisa que nos países onde o envelhecimento ocorreu mais lentamente, tanto os que exigem cuidados quanto seus cuidadores são contemplados por programas e políticas de Saúde Pública.

Pereira e Soares (2015), ao avaliar a qualidade de vida do cui- 
dador familiar do idoso com demência, salientam que o enfermeiro deve identificar as necessidades do cuidador para traçar estratégias com relação ao cuidado e melhorar a qualidade de vida. A sobrecarga dos cuidadores familiares de idosos dependentes é analisada por Lino et al (2016), que consideram que o apoio aos cuidadores e o tratamento da depressão e demência em seus cuidados, podem melhorar a qualidade de vida e saúde de ambos. A sobrecarga de cuidadores (geralmente familiares) e a dependência funcional de idosos é objeto da pesquisa de Gratão et al (2013), que defendem a prevenção e a intervenção precoce para a evitar sobrecarga. Necessidades sentidas e normativas dos cuidadores familiares são alvos da investigação de Bierhals et al (2017). As maiores necessidades sentidas estão ligadas a aspectos subjetivos do cuidado, enquanto as normativas dizem respeito as medicações. O papel do enfermeiro para compreender as necessidades do cuidador e planejar intervenções é acentuado.

A ampliação do número de cuidadores decorrente do envelhecimento populacional é alvo de três estudos distintos. Barros, Silva e Leite (2015) tratam do aumento de cuidadores de idosos, reforçando que administram medicamentos. Giacomin et al (2005) ressaltam os fatores relacionados à necessidade de cuidados entre idosos de uma cidade mineira e identificam que $23 \%$ dos idosos precisam de cuidadores. As autoras referem o cuidado dos idosos dependentes como "problema de saúde pública". Lima (2016) discute a diminuição do cuidado em programas estatais de Portugal, como decorrência da crise econômica e social. Tal diminuição acarreta um aumento das práticas informais de cuidado o que, para a autora, se torna fator de sustentabilidade na medida que contribui para enfrentamento de crise, seja econômica, pessoal ou política.

A (in)visibilidade de idosos e cuidadores é discutida em dois artigos. Woodward (2016) aponta a invisibilidade de brancos idosos e seus cuidadores remunerados, por meio de fotografias, memórias, notícias e documentários. Dentro de uma cadeia global, a autora destaca que o cuidado é exercido por mulheres não brancas e comumente não cidadãs dos países em que trabalham. Debert (2016) aponta que a visibilidade de idosos e cuidadores, "dupla de indesejáveis", tem promovido redefinições nas formas de dependência, além de dar 
"novos significados às relações familiares, às obrigações do Estado e à vida doméstica" (Debert, 2016, p. 129).

Um último artigo analisa o autocuidado. Borges e Seidl (2012) investigam as percepções e comportamentos de homens idosos com relação ao autocuidado, sublinhando que diferentes barreiras (econômicas, socioculturais, pessoais e nos serviços de saúde) comprometem os cuidados com a saúde do homem. A adoção de práticas saudáveis passa por estratégias educacionais.

Após a sistematização dos conteúdos dentro das temáticas elencadas, foram averiguadas as referências bibliográficas citadas pelo conjunto de artigos por meio do Gephi, software livre que permite análise e visualização de redes. Todavia, para facilitar a visualização e verificar as referências que conectam diferentes autores, foi considerado o grau de entrada 2 na formação da rede, de forma que somente as referências citadas duas vezes ou mais compõem a figura 2 .

Figura 2: Rede das referências bibliográficas (grau de entrada 2)

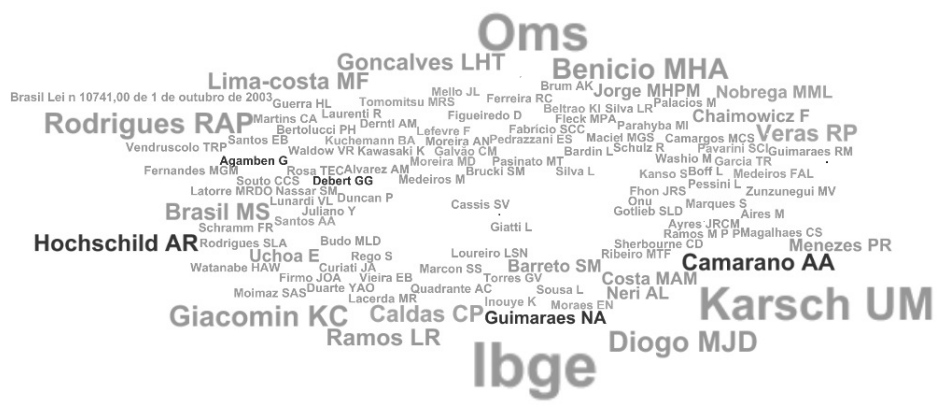

Fonte: Elaboração própria.

Ao todo 110 referências foram citadas por dois artigos ou mais, num universo que contabilizava mais de 1480 bibliografias. $\mathrm{O}$ Instituto Brasileiro de Geografia e Estatística (IBGE) e a Organização Mundial da Saúde (OMS/WHO) foram citados respectivamente por onze e nove dos artigos. Ursula Margarida Karsch foi referenciada por oito trabalhos. Maria Helena D’Aquino Benicio, Maria José 
D’Elboux Diogo, Karla Cristina Giacomin e Luiz Roberto Ramos foram citados em cinco. Ana Amélia Camarano, Arlie Russel Hoschild, Célia Pereira Caldas, Lucia Hisako Takase Gonçalves, Maria Fernanda Lima-Costa e Renato Peixoto Veras foram mencionados em quatro. A legislação brasileira foi referenciada em seis trabalhos, sendo quatro Ministério da Saúde (Brasil MS) e dois a Lei 10.741/2003, que versa sobre o Estatuto do Idoso.

Entre os autores mais referenciados, oito pertencem à grande área Ciências da Saúde (73\%), dois são das Ciências Sociais Aplicadas (18\%) e um das Ciências Humanas (9\%). Dos onze mais citados, somente três figuram como autores de dois artigos que compõem o corpus de análise desta pesquisa: Karsch (2003); Giacomin e Lima-Costa (Giacomin et al, 2005).

Com a formação da rede, foi possível visualizar como essas referências se distribuem e como aparecem organizadas por proximidade em quatro comunidades (Blondel et al, 2008). A maior comunidade - tem como evidência o IBGE e a OMS, e autores como Karsch, Benício, Diogo e Lima-Costa entre outros - aproxima discussões em torno do envelhecimento como direito e do envelhecimento saudável e ativo. Outra comunidade relaciona referenciais, com destaque para Veras e Caldas, que debatem condições de saúde da população idosa e políticas públicas (ou a falta delas) voltadas para o envelhecimento populacional. Uma terceira comunidade, onde não se sobressai nenhuma referência, parece unir estudos em torno da incapacidade funcional de idosos. A quarta comunidade, com destaque para Camarano e Hochschild, aproxima estudos acerca de diversas questões (sociais, econômicas e culturais), provenientes da redefinição demográfica.

É interessante observar que não há um autor que exerça centralidade como referencial obrigatório para pensar o cuidado nas políticas direcionadas ao envelhecimento, posto que a mais citada, Karsch, foi referenciada em oito trabalhos. Os outros mais citados foram utilizados por cinco artigos ou menos. Ou seja, há uma dispersão de referências, o que indica que vários autores são utilizados para discutir questões relacionadas à temática.

Em que pese o predomínio de autores da área de Ciências da 
Saúde entre as principais referências, nota-se a presença de outras áreas de conhecimento, como Ciências Sociais aplicadas e Ciências Humanas. A própria Karsch é do Serviço Social. Isso indica que, embora a maioria dos autores mais referenciados seja da Saúde, outras áreas também participam do debate e da produção de conhecimento que compreende o cuidado como fundamental nas políticas públicas voltadas para o envelhecimento. Não poderia ser diferente, posto que o cuidado revela uma miríade de sentimentos e ações (Tronto, 1997), é constitutivo do ser (Boff, 2012), envolve diferentes dimensões da vida, além da técnica, e não é restrito à área da Saúde (Leininger, 2000, Waldow, 2006).

\section{CONSIDERAÇÕES FINAIS}

Este trabalho objetivou analisar o cuidado na produção de conhecimento de políticas públicas voltadas para o envelhecimento disponível no SciELO, em periódicos altamente qualificados em Sociologia. Por meio de um exercício bibliométrico analisou autores e periódicos, com suas respectivas vinculações institucionais, bem como o conteúdo dos artigos a partir dos temas, resumos, palavras-chave e referências, elencando as seguintes temáticas: cuidado em Instituições de Longa Permanência; cuidado em Enfermagem; cuidado/cuidador familiar; ampliação do número de cuidadores; invisibilidade de idosos e cuidadores; cuidado no final da vida; e, autocuidado. Verificou, por um lado, que a maior parte dos artigos estão relacionados à grande área das Ciências da Saúde e são publicados na região sudeste, que concentra importantes instituições de ensino e pesquisa. Por outro, constatou que o cuidado, assim como as políticas públicas, é um tema interdisciplinar, central para pensar o envelhecimento, e que é abordado também em outras áreas de conhecimento, como as Ciências Humanas e Sociais e as Ciências Sociais Aplicadas.

Apesar da diversidade das pesquisas, elas têm em comum a compreensão de que o cuidado é essencial no processo de envelhecimento. Chama a atenção a feminização do cuidado, recorrente em vários artigos que analisam tanto o cuidado profissional/formal 
quanto o cuidado familiar/informal. As mulheres são cuidadoras na maioria dos cenários, seja no mundo do trabalho ou na esfera doméstica, onde estão sobrecarregadas. Considerando que a expectativa de vida da mulher é maior, as mulheres são também objeto de cuidado na velhice. E, no caso desta pesquisa, as mulheres são as principais autoras dos artigos analisados. Em outros termos, há uma feminização do envelhecimento, do trabalho do cuidado e da abordagem do cuidado na produção acadêmica sobre políticas de envelhecimento.

A corresponsabilidade do Estado e da sociedade no cuidado aos idosos também merece destaque, apesar da família continuar exercendo papel central nesse processo. A legislação voltada para o envelhecimento no Brasil, a exemplo das leis 8.842/1994 e $10.741 / 2003$, destaca que cabe a essas três esferas garantir ao idoso o direito de envelhecer com dignidade. Nesse sentido, muitos dos artigos que compõem o corpus de análise sublinham a importância do papel do Estado e apontam a necessidade de políticas públicas efetivas acerca do processo de envelhecimento. Outros abordam o cuidado familiar, que parece seguir predominando na velhice apesar do crescimento das instituições de longa permanência, e apontam a importância de o cuidador também ser atendido/acolhido por políticas e serviços de saúde. Num país que envelhece rapidamente, é fundamental que o Estado e a sociedade assumam também na prática sua responsabilidade na ação de cuidar de seus idosos, atendendo velhas demandas e se preparando para novos desafios.

Por fim, o exercício bibliométrico aqui realizado, acompanhado análise de redes de referências bibliográficas, se mostrou viável para pesquisas com um número de artigos bem maior, além de instigar novas possibilidades de pesquisa e reflexões sobre a temática do envelhecimento, fundamental diante do atual cenário. Uma delas, em andamento, é a análise da produção sobre políticas públicas direcionadas ao envelhecimento populacional. Outra, já mencionada nas notas metodológicas, se refere à produção do conhecimento sobre cuidado, entendendo-o como algo amplo, que envolve trabalho e suscita emoções, e circula de diferentes formas na vida social. 
O cuidado como objeto de pesquisa na produção de conhecimento sobre políticas públicas para o envelhecimento.

\section{REFERÊNCIAS}

BARDIN, L. Análise de conteúdo. São Paulo: Edições 70, 2011.

BOFF, L. Saber cuidar: Ética do humano e compaixão pela terra. Petrópolis: Vozes, 2004.

BRASIL. Constituição da República Federativa do Brasil. Brasília, DF, 1998. Disponível em: www.planalto.gov.br/ccivil_03/constituicao/ constituicaocompilado.htm. Acesso em 08/01/2018.

BRASIL. Presidência da República. Lei 8.842, de 04 de janeiro de 1994. Dispõe sobre a política nacional do idoso, cria o Conselho Nacional do Idoso e dá outras providências. Diário Oficial da União. Brasília, DF, 05 jan. 1994. Disponível em: http://www.planalto.gov.br/ ccivil_03/leis/L8842.htm Acesso em 21/03/2017.

BRASIL. Decreto $n^{\circ} 1.948$, de 03 de julho de 1996. Regulamenta a Lei $\mathrm{n}^{\circ}$ 8.842, de 04 de janeiro de 1994, que dispõe sobre a Política Nacional do Idoso e dá outras providências. Disponível em: http://www. planalto.gov.br/ccivil_03/decreto/d1948.htm. Acesso em 21/03/2017.

BRASIL. Presidência da República. Lei 10.741, de $1^{\circ}$ de outubro de 2003. Dispõe sobre o Estatuto do Idoso e dá outras providências. Diário Oficial da União. Brasília, DF, 03 out. 2003. Disponível em: http:// www.planalto.gov.br/ccivil_03/leis/2003/L10.741.htm Acesso em 14/03/2017.

BLONDEL, V. D. et al. Fast unfolding of communities in large networks. Journal of Statistical Mechanics: Theory and Experiment, vol. 2, 2008. Disponível em: http://iopscience.iop. org/article/10.1088/1742-5468/2008/10/P10008/meta Acesso em $10 / 08 / 2018$.

CAMARANO, A. A.; PASINATO, M. T. O envelhecimento populacional na agenda das políticas públicas. In: CAMARANO, A. A. (Org.). Os novos idosos brasileiros: muito além dos 60. Rio de Janeiro: IPEA, 2004, p. 261-300.

CAMARANO, A. A. O Estatuto do Idoso: avanços com contradições. Textos para discussão. Brasília, Rio de Janeiro, IPEA, 2013.

CLEMENTE, A. J.; JULIANO, M. C. A produção do conhecimento em cidadania no Brasil: uma análise a partir do SciELO (1992-2011). Tomo, Vol. 23, nº. 2, pp. 173-216, 2013.

COLLIÈRE, M. F. Promover a vida: da prática das mulheres de virtude aos cuidados de enfermagem. Lisboa: Lidel, 1999. 
CONNER, N.; PROVEDEL, A.; MACIEL, E. L. N. Ciência \& Saúde Coletiva: análise da produção científica e redes colaborativas de pesquisa. Ciênc. saúde coletiva. Vol. 22, nº.3, pp.987-996, 2017.

DEBERT, G.G. A reinvenção da velhice: Socialização e processos de reprivatização do envelhecimento. São Paulo: Fapesp, 1999.

HIRATA, H.; GUIMARÃES, N. A. Cuidado e cuidadoras. As várias faces do trabalho do care. São Paulo: Atlas, 2012.

FRANCISCO, E. R. RAE-eletrônica: exploração do acervo à luz da bibliometria, geoanálise e redes sociais. RAE-eletrônica, Vol. 51, no.3, pp. 280-306, 2011. Disponível em: http://www.scielo. br/scielo.php?pid=S0034-75902011000300008\&script $=$ sci abstract\&tlng=pt Acesso em 10/08/2018.

GAWANDE, A. Mortais: nós, a medicina e o que realmente importa no final. Rio de Janeiro: Objetiva, 2015.

IBGE. Tábua completa de mortalidade para o Brasil - 2016. Rio de Janeiro, 2017. Disponível em:ftp://ftp.ibge.gov.br/Tabuas_Completas_de Mortalidade/Tabuas_Completas_de_Mortalidade_2016/tabua_de_ mortalidade_2016_analise.pdf. Acesso em: 17/07/2018.

IBGE. Síntese de indicadores sociais. Rio de Janeiro, 2015. Disponível em: http://biblioteca.ibge.gov.br/visualizacao/livros/liv95011.pdf Acesso em 02/01/2017

LEININGER, M. Teoria da Enfermagem transcultural. In: GEORGE, J. B. et al. Teorias de Enfermagem: os fundamentos à prática profissional. Porto Alegre: Artmed, 2000.

LOPES, M.J.M.; LEAL, S.M.C. A feminização persistente na qualificação profissional da enfermagem brasileira. Cadernos Pagu, Campinas, no. 24, pp. 105-125, 2005.

METZ, T.; JÄCKLE, S. Patterns of publishing in political science journals: An overview of our profession using bibliographic data and a coauthorship network. PS: Political Science \& Politics, Vol. 50, nº. 1, pp. 157-165, 2017.

TRONTO, J. Mulheres e cuidados: o que as feministas podem aprender sobre a moralidade a partir disso? In: JAGGAR, A. M.; BORDO, S. R. Gênero, Corpo, Conhecimento. Rio de Janeiro: Record, 1997.

UNFPA. Fundo de População das Nações Unidas. Envelhecimento no Século XXI: Celebração e Desafios. 2012. Disponível em: https:// www.unfpa.org/sites/default/files/pub-pdf/Portuguese-ExecSummary_0.pdf. Acesso em 29/05/2018. 
O cuidado como objeto de pesquisa na produção de conhecimento sobre políticas públicas para o envelhecimento.

WALDOW, V. R. Cuidar - Expressão humanizadora da enfermagem. Petrópolis: Vozes, 2006.

WHO. Envelhecimento ativo: uma politica de saúde. Brasília: Organização Pan-Americana da Saúde, 2005. Disponível em: http://bvsms.saude. gov.br/bvs/publicacoes/envelhecimento_ativo.pdf Acesso em: 29/05/2018.

\section{APÊNDICE - CORPUS DE ANÁLISE}

BARBOSA, L. M. et al. Qualidade de vida relacionada à saúde dos cuidadores formais de idosos institucionalizados em Natal, Rio Grande do Norte. Rev. bras. est. popul. São Paulo, Vol. 34, nº. 2, pp.391-414, 2017.

BARROS, D. S. L.; SILVA, D. L. M.; LEITE, S. N. Conduta do tratamento medicamentoso por cuidadores de idosos. Interface (Botucatu). Botucatu, Vol. 19, no. 54, pp.527-536, 2015.

BATISTA, A. S.; ARAÚJO, A. B. Intimidade e mercado: o cuidado de idosos em instituições de longa permanência. Soc. Estado, Brasília, Vol. 26, no. 1, pp. 175-195, 2011.

BATISTA, A. S.; BANDEIRA, L. M. Trabalho de cuidado: um conceito situacional e multidimensional. Rev. Bras. Ciênc. Polít., Brasília, nº 18, pp. 59-80, 2015.

BIERHALS, C. C. B. K. et al. Necessidades dos cuidadores familiares na atenção domiciliar a idosos. Rev. Latino-Am. Enfermagem, Ribeirão Preto, Vol. 25, e2870, 2017.

BORGES, L. M.; SEIDL, E. M. F. Percepções e comportamentos de cuidados com a saúde entre homens idosos. Psicol. ciênc. prof., Brasília, Vol. 32, nº 1, pp. 66-81, 2012.

CALDEIRA, S. et al. O enfermeiro e o cuidado à mulher idosa: abordagem da fenomenologia social. Rev. Latino-Am. Enfermagem, Ribeirão Preto, Vol. 25, no. 5, pp. 888-895, 2012.

CARVALHAIS, M.; SOUSA, L. Qualidade dos cuidados domiciliares em enfermagem a idosos dependentes. Saúde e Sociedade, São Paulo, Vol. 22, no. 1, pp.160-172, 2013.

DEBERT, G. G. Migrações e o Cuidado do idoso. Cad. Pagu, Campinas, nº. 46, pp. 129-149, 2016. 
DEL DUCA, G. F.; MARTINEZ, A. D.; BASTOS, G. A. N. Perfil do idoso dependente de cuidado domiciliar em comunidades de baixo nível socioeconômico de Porto Alegre, Rio Grande do Sul. Ciênc. saúde coletiva, Rio de Janeiro, Vol. 17, nº 5, pp. 1159-1165, 2012.

FREITAS, A. V. da S.; NORONHA, C. V. Idosos em instituições de longa permanência: falando de cuidado. Interface (Botucatu). Botucatu, Vol. 14, n'. 33, pp. 359-369, 2010.

GIACOMIN, K. C. et al. Projeto Bambuí: um estudo de base populacional da prevalência e dos fatores associados à necessidade de cuidador entre idosos. Cad. Saúde Pública, Rio de Janeiro, Vol. 21, nº. 1, pp. 80-91, 2005.

GOMES, A. L. Z.; OTHERO, M. B. Cuidados paliativos. Estud. Av., São Paulo, Vol. 30, no. 88, pp. 155-166, 2016.

GRATÃO, A. C. M. et al. Dependência funcional de idosos e a sobrecarga do cuidador. Rev. esc. enferm. USP, São Paulo, Vol. 47, nº.1, pp. 137-144, 2013.

ISRAEL, N. E.N.; ANDRADE, O. G. de; TEIXEIRA, J. J. V. A percepção do cuidador familiar sobre a recuperação física do idoso em condição de incapacidade funcional. Ciênc. saúde coletiva, Rio de Janeiro, Vol. 16 (suppl. 1), pp. 1349-1356, 2011.

KARSCH, U. M. Idosos dependentes: famílias e cuidadores. Cad. Saúde Pública, Rio de Janeiro, Vol.19, nº 3, pp. 861-866, 2003.

KÜCHEMANN, B. A. Envelhecimento populacional, cuidado e cidadania: velhos dilemas e novos desafios. Soc. Estado, Brasília, Vol. 27, nº. 1, pp. 165-180, 2012.

LIMA, A. P. de. O cuidado como elemento de sustentabilidade em situações de crise. Portugal entre o Estado providência e as relações interpessoais. Cad. Pagu, Campinas, n. 46, pp. 79-105, 2016.

LINO, V. T. S. et al. Prevalência de sobrecarga e respectivos fatores associados em cuidadores de idosos dependentes, em uma região pobre do Rio de Janeiro, Brasil. Cad. Saúde Pública, Rio de Janeiro, Vol. 32, n'. 6, 2016.

MEIRELES, V. C. et al. Características dos idosos em área de abrangência do Programa Saúde da Família na região noroeste do Paraná: contribuições para a gestão do cuidado em enfermagem. Saúde e Sociedade, São Paulo, Vol.16, nº.1, pp. 69-80, 2007.

MELLO, A. L. S. F. de; ERDMANN, A. L. Revelando contradições e incorporando melhores práticas no cuidado à saúde bucal de idosos. 
Physis, Rio de Janeiro, Vol. 17, nº. 1, pp. 139-156, 2007.

MENDES, J.; SOARES, V. M. N.; MASSI, G. A. A. Percepções dos acadêmicos de fonoaudiologia e enfermagem sobre processos de envelhecimento e a formação para o cuidado aos idosos. Revista CEFAC, 17(2), 576-585, 2015.

PEREIRA, L. S. M.; SOARES, S. M. Fatores que influenciam a qualidade de vida do cuidador familiar do idoso com demência. Ciênc. saúde coletiva, Rio de Janeiro, Vol. 20, no. 12, pp. 3839-3851, 2015.

PIMENTA, G. M. F.et al. Perfil do familiar cuidador de idoso fragilizado em convívio doméstico da grande Região do Porto, Portugal. Rev. esc. enferm. USP, São Paulo, Vol. 43, n’. 3, pp. 609-614, 2009.

QUEIROZ, A. H. A. B. et al. Percepção de familiares e profissionais de saúde sobre os cuidados no final da vida no âmbito da atenção primária à saúde. Ciênc. saúde coletiva, Rio de Janeiro, Vol. 18, $\mathrm{n}^{\circ}$. 9, pp. 2615-2623, 2013.

RESENDE, M. C. F.; DIAS, E. C. Cuidadores de idosos: um novo / velho trabalho. Physis, Rio de Janeiro, Vol. 18, no. 4, pp. 785-800, 2008.

RIBEIRO, M. T. de F. et al. Perfil dos cuidadores de idosos nas instituições de longa permanência de Belo Horizonte, MG. Ciênc. saúde coletiva, Rio de Janeiro, Vol. 13, no. 4, pp. 1285-1292.

ROSAS, R. E. Feminización y colectivización del cuidado a la vejez en México. Cad. Pesqui., São Paulo, Vol. 44, no. 152, pp. 378-399, 2014.

WOODWARD, K. Um segredo público: o viver assistido, cuidadores, globalização. Cad. Pagu, Campinas, no. 46, pp. 17-57, 2016. 